\title{
EXTRACTING RADIAL VELOCITIES OF A- AND B-TYPE STARS FROM ECHELLE SPECTROGRAPH CALIBRATION SPECTRA
}

\author{
Juliette C. Becker ${ }^{1,2,5}$, John Asher Johnson ${ }^{3,6}$, Andrew Vanderburg ${ }^{3,5}$, and Timothy D. Morton ${ }^{4}$ \\ ${ }^{1}$ Department of Astronomy, University of Michigan, 1085 South University Avenue, Ann Arbor, MI 48109, USA; jcbecker@umich.edu \\ ${ }^{2}$ Cahill Center for Astronomy and Astrophysics, California Institute of Technology, 1200 East California Boulevard, Pasadena, CA 91125, USA \\ ${ }^{3}$ Harvard-Smithsonian Center for Astrophysics, 60 Garden Street, Cambridge, MA 02138, USA \\ ${ }^{4}$ Department of Astrophysical Sciences, 4 Ivy Lane, Peyton Hall, Princeton University, Princeton, NJ 08544, USA \\ Received 2014 November 3; accepted 2015 March 12; published 2015 April 15
}

\begin{abstract}
We present a technique to extract radial velocity (RV) measurements from echelle spectrograph observations of rapidly rotating stars $\left(V \sin i \gtrsim 50 \mathrm{~km} \mathrm{~s}^{-1}\right)$. This type of measurement is difficult because the line widths of such stars are often comparable to the width of a single echelle order. To compensate for the scarcity of lines and Doppler information content, we have developed a process that forward-models the observations, fitting the RV shift of the star for all echelle orders simultaneously with the echelle blaze function. We use our technique to extract RV measurements from a sample of rapidly rotating A- and B-type stars used as calibrator stars observed by the California Planet Survey observations. We measure absolute RVs with a precision ranging from $0.5-2.0 \mathrm{~km} \mathrm{~s}^{-1}$ per epoch for more than 100 A- and B-type stars. In our sample of 10 well-sampled stars with RV scatter in excess of their measurement uncertainties, three of these are single-lined binaries with long observational baselines. From this subsample, we present detections of two previously unknown spectroscopic binaries and one known astrometric system. Our technique will be useful in measuring or placing upper limits on the masses of sub-stellar companions discovered by wide-field transit surveys, and conducting future spectroscopic binarity surveys and Galactic space-motion studies of massive and/or young, rapidly rotating stars.
\end{abstract}

Key words: binaries: general - methods: data analysis - techniques: radial velocities

\section{INTRODUCTION}

Stellar radial velocity (RV) measurements have become increasingly precise over the past $30 \mathrm{yr}$ due to the advent and development of high-resolution spectrographs equipped with digital detectors (Campbell et al. 1981), including HIRES at Keck (Vogt et al. 1994; Howard et al. 2010); particularly with the construction of environmentally stabilized spectrometers such as the HARPS-South and -North spectrographs (Mayor et al. 2003; Cosentino et al. 2012), SOPHIE at Haute-Provence (Bouchy et al. 2009), CHIRON at CTIO (Schwab et al. 2010), and the Planet Finder Spectrograph (PFS) at Magellan (Crane et al. 2006, 2010). While the discovery and characterization of exoplanets has been the driving scientific motivation behind these developments (e.g., Mayor \& Queloz 1995; Butler et al. 1999, 2004; Dumusque et al. 2012), increased measurement precision has also led to significant advances in understanding stellar binarity, particularly around Sun-like stars (Duquennoy \& Mayor 1991; Fischer \& Marcy 1992; Raghavan et al. 2010).

However, the stability of a given spectrometer is only part of what enables high RV precision. The attainable Doppler precision also depends greatly on the type of star observed. Measurements at the highest attainable precision today, levels at or below $1 \mathrm{~m} \mathrm{~s}^{-1}$, can only be performed on stars with spectra that contain many sharp spectral lines. As a result, most RVbased planet surveys have been restricted to F-, G-, K-, and Mtype dwarf stars, which rotate slowly and display numerous fine spectral features.

On the other hand, more massive A- and B-type stars have hotter atmospheres and exhibit fewer absorption features. Also,

\footnotetext{
5 NSF Graduate Research Fellow.

${ }^{6}$ David \& Lucile Packard Fellow.
}

because these hot stars lack convective outer layers, they retain most of their primordial angular momentum, and what few spectral features they show are highly rotationally broadened. For these reasons, rapidly rotating hot and massive stars have nearly featureless blackbody spectra, showing only very broad hydrogen and helium absorption lines, as illustrated in Figure 1. Rotational smearing also affects young stars of all masses if they have not yet lived long enough to have experienced sufficient magnetic braking. It is thus much more challenging to obtain precise RVs for rapidly rotating stars from highresolution echelle observations.

At the same time, their nearly featureless spectra make hot stars excellent calibrators for measuring and removing telluric absorption features, and as calibrators for Doppler surveys (as well as for instrumental tests, as in Spronck et al. 2013). These "blackbodies in the sky" are excellent calibrators of the transmission functions of absorption cells used as wavelength references, and as means of measuring the spectrometer's instrumental profile for surveys using gas absorption cells. As a result, there exists a large library of high-resolution spectra of hot stars obtained as calibrators of high-precision, gas-cell calibrated Doppler surveys such as the California Planet Survey (CPS).

While this library was obtained for calibration purposes rather than as a scientific data product, it serendipitously provides the opportunity to conduct an RV survey of hot stars. Multiplicity studies of high mass stars are important to constrain models of their formation (Bonnell \& Bate 2005; Zinnecker \& Yorke 2007). Some notable massive-star RV studies include those of Galland et al. (2005), who studied the multiplicity of A- and F-type dwarfs with rapid rotation rates, Chini et al. (2012), who examined the binary fraction among B- and O-type stars, and Huang et al. (2010), who examined 
RVs as a larger-scale effort to measure the projected rotational velocities of massive stars.

In addition to binarity surveys, absolute RVs (velocities measured with respect to the solar system barycenter) offer the ability to study the local motion and bulk flow of stars in the Galaxy, informing cluster dynamics, providing formation insights to the formation histories of visible stars, and providing information about the assembly of the Milky Way and studies of open clusters (e.g., Mermilliod et al. 2009; Frebel 2010). Absolute RVs are also needed to calibrate other measurements. For example, the Hipparcos-Gaia HundredThousand Proper-Motion survey, which aims to find the proper motions for over $\sim 10^{5}$ stars over a 23 yr baseline, requires RV measurements of its target stars to account for acceleration that might be affecting the proper motion measurements (de Bruijne \& Eilers 2012).

While echelle spectra of rapidly rotating A- and B-type stars show very few absorption features, the high signal-to-noise ratio $(\mathrm{S} / \mathrm{N})$ and the highly oversampled nature of the spectral features of their spectra should, in principle, provide RVs better than $1 \mathrm{~km} \mathrm{~s}^{-1}$ (see Appendix A). This precision allows both
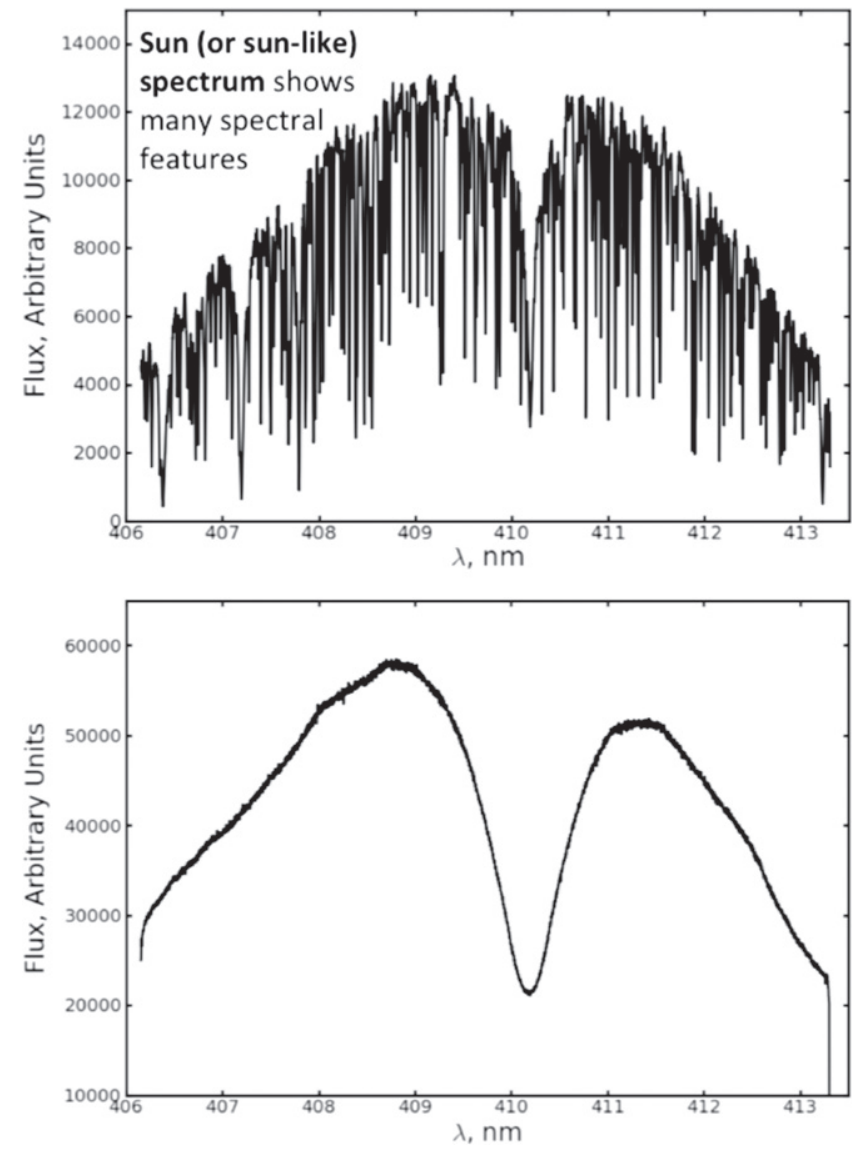

Figure 1. Radial velocity measurements using A- and B-type stellar spectra are hindered by rotational broadening of their observed spectral features. Here, we see this effect as illustrated by the $H \delta 410.1 \mathrm{~nm}$ Balmer line. Upper panel: one HIRES echelle order of the Solar spectrum measured by observing reflected sunlight from the asteroid Vesta. This spectrum is representative of those of low-mass stars observed by the CPS program. Lower panel: the same HIRES order, this time showing an observation of an A-type star, HR 6827. This rapidly rotating star has hundreds of times fewer spectral features than are seen in the Solar spectrum. The high-mass star cannot be analyzed in the same way as a Sun-like due to its broad spectral features, which are significant fraction of their echelle orders. absolute measurements to measure the space motions of these bright stars, as well as relative RV measurements to search for binary companions.

In Section 2 we present a new analysis technique to extract RV measurements from echelle spectra of rapidly rotating stars, for both differential (Section 2.4) and absolute (Section 2.5) velocities. Section 3 presents the results of applying our method to a large number of archival high-resolution spectra of A- and B-type stars. These observations were obtained for use as calibrators by the California Planet Survey RV planet search program at Keck Observatory, over a span of $8 \mathrm{yr}$ since the HIRES detector upgrade. We achieve a typical precision of $1 \mathrm{~km} \mathrm{~s}^{-1}$, and recover the orbital motion of several known astrometric or spectroscopic binaries. Additionally, we detect long-term RV trends for two stars (HR 5867 and HR 8028) and spectroscopically confirm the astrometric binary HR 3067.

\section{OBSERVATIONS AND ANALYSIS}

\subsection{Data Collection}

The data presented herein were collected with the HIRES on the Keck I telescope (Vogt et al. 1994). HIRES was operated in the standard CPS observing mode with the red-optimized grating with a spectral resolving power of $\Delta \lambda / \lambda \approx 55,000$. Across the $8 \mathrm{yr}$ of observations, various slit masks, or "deckers" were used, including C1, C5, B1 and B5. ${ }^{7}$ Because the targets are so bright and since the rotational broadening of the stars is large enough that all features are resolved, the different observing modes have little effect on the final results.

HIRES has three charge coupled devices (CCDs), each of which covers a different wavelength range of the spectrum. Colloquially, these CCDs are referred to as the blue $(364.3-479.5 \mathrm{~nm})$, green $(497.7-642.1 \mathrm{~nm})$, and red $(654.3-799 \mathrm{~nm})$ chips. These CCDs have twenty-three, sixteen, and ten orders, respectively, with each constituent order containing 4020 pixels. We reduce the HIRES CCD images using the standard CPS method of using an optimal extraction technique to trace spectral orders on the twodimensional echelle image, rectifying the orders, and then summing pixels in columns to obtain a one-dimensional spectrum for each order.

The observations of rapidly rotating calibrators were often, but not always, made with the iodine cell in the light path in order to measure the instrumental profile (line-spread function) from the sharp iodine absorption features (Butler et al. 1996; Johnson et al. 2006). In our analysis, we take advantage of the simultaneous iodine reference to determine the spectrograph's wavelength solution. In the cases of exposures taken without the iodine cell, we search for the nearest observation in time taken with the iodine cell and use its wavelength solution instead. We note, however, that the region containing iodine lines only spans about $100 \mathrm{~nm}$ about on the green chip between roughly $500 \mathrm{~nm}$ and $600 \mathrm{~nm}$, and the majority of the spectral features characteristic of hot, rapidly rotating stars (in particular, hydrogen and helium lines) are located on the blue chip. Using the iodine-derived wavelength solution requires extrapolation to the rest of the HIRES bandpass, which we describe in Section 2.2.

http://www2.keck.hawaii.edu/inst/hires/manual2.pdf 


\subsection{Wavelength Solution}

HIRES is not an environmentally stabilized instrument like other precise RV instruments, so the spectrograph's wavelength solution drifts over the course of a night at the level of $1-2 \mathrm{~km} \mathrm{~s}^{-1}$ (roughly a pixel). The CPS program circumvents this problem by passing starlight through an iodine gas cell, which imprints a wavelength reference spectrum onto the intrinsic stellar spectrum (Valenti et al. 1995). The simultaneous iodine reference permits wavelength solutions to a precision better than a fraction of $\mathrm{a} \mathrm{m} \mathrm{s}^{-1}$, significantly more precise than is necessary for RV measurements of rapidly rotating hot stars. Unfortunately, this wavelength solution is only measurable between roughly 500 and $600 \mathrm{~nm}$, where there is significant iodine absorption and a lack of strong telluric absorption features.

We found that even though the iodine wavelength solution was only calculated over a small region, it was possible to extrapolate the wavelength solution to other spectral regions with a precision of better than $400 \mathrm{~m} \mathrm{~s}^{-1}$. This is possible because the orientation of the three CCDs is such that all orders (on all three chips) are parallel, with the response functions by pixel remaining consistent (to $0.4 \mathrm{~km} \mathrm{~s}^{-1}$ ) between orders. The distance between chips is only 6-7 pixels $^{8}$, so the wavelength mapping for one CCD is closely matched by the neighboring CCDs.

We fit the wavelength solution from the iodine region on the green chip with the following model:

$$
\lambda_{\text {extrap }}(i, n)=A+B \times i+C \times i^{2}+D \times n
$$

where $n$ is the order number, $i$ is the pixel number in the dispersion direction, and $A, B, C$, and $D$ are the fitted coefficients. This model does a good job of describing the dependence in the dispersion direction, but the simple linear dependence of wavelength on order number is only adequate to describe the wavelength solution to a precision of $\simeq 100 \mathrm{~m} \mathrm{~s}^{-1}$ on the green chip. Fitting only a linear dependence on order number, however, allows us to extrapolate the wavelength solution without the problem of a higher order polynomial fit diverging quickly. For an example wavelength solution, shown in Figure 2, the nominal solution must be corrected by values varying by $400 \mathrm{~m} \mathrm{~s}^{-1}$ between the bluest and reddest orders. The exact value of this deviation varies by observation, but the values given here are typical values. The correction computed from the green chip can then be applied to the blue chip, resulting in a wavelength solution with at least $400 \mathrm{~m} \mathrm{~s}^{-1}$ better precision between orders on the far edges of the chip and $1.4 \mathrm{~km} \mathrm{~s}^{-1}$ better precision compared to the nominal wavelength solution for the entire run.

We show the result of one of the fits in Figure 2 and compare the fit to the data in the iodine region. The uncorrected dependence on order number is evident, but the typical errors introduced are small. Due to the large drifts in the wavelength solution of the spectrograph, the extrapolated solution is of higher quality in general than the nightly solution, $\left(\lambda_{\text {nightly }}\right)$, which is calculated from a Thorium-Argon lamp exposure at the beginning of the night. We therefore use the extrapolated wavelength solutions in our analysis hereafter.

\footnotetext{
http://www2.keck.hawaii.edu/inst/hires/hires_data.pdf
}

\subsection{Continuum Shape}

Unlike traditional Doppler techniques developed for F-, G-, $\mathrm{K}-$, and M-stars, which perform analysis on continuum normalized spectra, the peculiarities of our hot, rapidly rotating stellar sample require us to simultaneously fit for RVs with the spectrograph's blaze function. Hot, rapidly rotating stars like those considered in this study have very broad spectral features, some of which have line widths that are a significant fraction $(\sim 10-20 \%)$ of the width of a HIRES echelle order (typically $5 \mathrm{~nm}$ in the blue). Ignoring this would introduce biases caused by the degeneracy between the overall flux level and the location of spectral lines, preventing effective normalization to the continuum.

The shape of the continuum for each spectral order in echelle spectrographs is a blaze function determined by the spacing of grooves on the diffraction grating. The Fourier transform of the shape of each groove on the grating results in a sinc $(\sin (x) / x)$ function, the first maximum of which is known as the blaze function. The shapes of the spectral orders are similar due to their common physical origin, but other spectrograph optical effects result result in small changes between orders.

We take advantage of the fact that the echelle orders have similar blaze function shapes when modeling them in our RV fits, and model the continuum shape of the continuum as a function of both order number and pixel number in the dispersion direction. Essentially, the continuum level of each spectral order is a slice from one continuous, three-dimensional function $F(i, n)$, where $F$ is the flux level, $n$ is to the order number, and $i$ is to the pixel number in the dispersion direction.

We experimented with various functional forms for $F(i, n)$ by fitting to flat field exposures - that is, calibration exposures taken when the HIRES slit was illuminated with a quartz lamp continuum source. We settled on the following form for $F(i, n)$ :

$$
F(i, n)=c_{0} i^{2}+c_{1} i+c_{2}+c_{3} n+c_{4} n^{2}+c_{5} i n,
$$

where the coefficients, $\left\{c_{j}\right\}$, are free parameters.

\subsection{Fitting Procedure}

We measure RVs by simultaneously fitting a model to the continuum shape and Doppler shift of each spectrum. We start by selecting the first observation for a given star and set this spectrum to be our stellar template spectrum, analogous to the deconvolved intrinsic stellar spectrum used in iodine cell Doppler analysis (Butler et al. 1996). However, since the instrumental line-broadening is negligible to the rotational broadening, no deconvolution is required.

We then use a Levenberg-Marquardt (Press et al. 2002) least squares technique to find the best-fitting Doppler shift for each observation of a particular star. The Levenberg-Marquardt algorithm is relatively robust, but can sometimes get stuck in local extrema in the function it is minimizing or maximizing, so we take care to find good initial guesses for the fit parameters. We first estimate the Doppler shift by performing a cross correlation between the template spectrum with the observation on one particular spectral order, the one containing the $\mathrm{H}-\gamma$ line at $434.047 \mathrm{~nm}$. We estimate the shape of the continuum using our fits to the flat field lamp as described in Section 2.3. Once we have initial guesses, we perform the Levenberg-Marquardt maximization on the following log-likelihood (where 


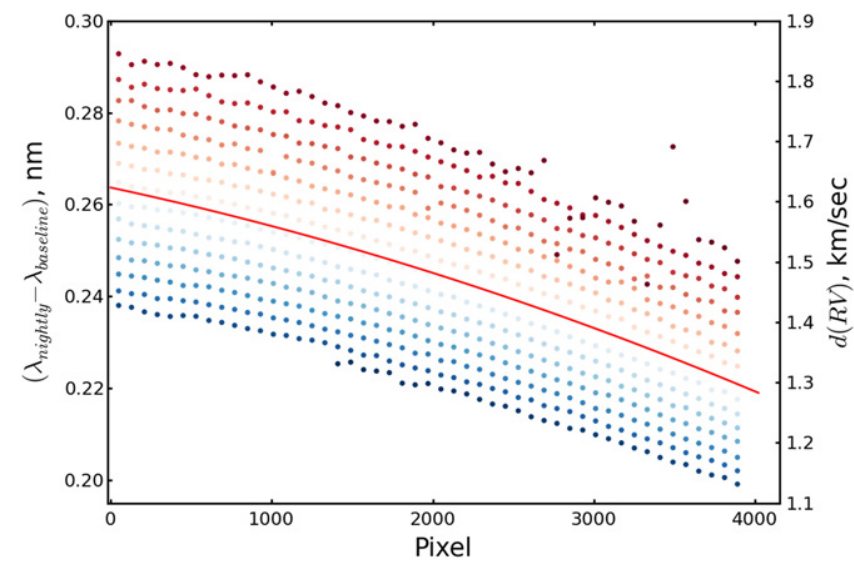

Figure 2. Difference between HIRES wavelength solution, $\lambda_{\text {nightly }}$, from one epoch compared to the nominal wavelength solution from an arbitrary reference epoch as a function of pixel and order number for the green chip. Each order is represented by a different color, from the bluest to reddest order. The dots are measurements of $\delta \lambda$, the deviation in wavelength from the nominal solution, and the solid line is the best-fitting solution as described by Equation (1) for an arbitrary order $n$. The offset from zero indicates that the spectrograph's wavelength zero-point has shifted from the nominal value by about $1.4 \mathrm{~km} \mathrm{~s}^{-1}$ at this epoch, and the slope-wavelength zeropoint as a function of pixel-is described by coefficients $B$ and $C$ in Equation (1).

$\left.\ln L \propto-\chi^{2}\right)$ function:

$$
\ln L=\sum_{i=0}^{N-1}\left[-\frac{1}{2} \ln \left(2 \pi \sigma_{i}\right)-\frac{1}{2}\left(\frac{\mathcal{I}_{i}-\mathcal{I}_{m}}{\sigma_{i}}\right)^{2}\right]
$$

where $\sigma_{i}$ is the error in flux on each pixel, $N$ is the total number of pixels, and $\mathcal{I}_{i}$ is the flux of the datum spectrum at pixel $i$. The model $\mathcal{I}_{m}$ is given by the following expression:

$$
\mathcal{I}_{m}(i, n)=F_{\text {ratio }}(i, n) \cdot S_{m}\left[\lambda_{\text {extrap }}(i, n)\left(1+\frac{V_{\text {Dop }}}{c}\right)\right]
$$

where $i$ is pixel number, $n$ is order number, $F_{\text {ratio }}(i, n)$ is the ratio between two continuum levels which share the functional form of Equation (2), and $S_{m}[i, n]$ is the original flux level of the first observed spectrum, which is used as the model. It is not necessary to include the convolution kernel in the fit for relative $\mathrm{RVs}$, as the rotation rate and instrument profile are expected to be constant between successive observations. If desired, the broadening can be fit by convolving Equation (4) with the kernel as described in Equation (6).

After the likelihood maximization, we extract the best-fitting Doppler shift parameter $\left(V_{\text {Dop }}\right)$. We treat the other model inputs as nuisance parameters. Finally, we apply a barycentric correction to the best-fitting Doppler shift to correct for the Earth's motion with respect to the target star. The barycentric correction depends primarily on the declination of the target, reaching a maximum for targets on the ecliptic. Higher-order contributions to the barycentric correction can be safely ignored at our target precision of $\sim 1 \mathrm{~km} \mathrm{~s}^{-1}$. We computed the barycentric correction with a python adaptation of the baryvel code (Stumpff 1980) with errors much smaller than our expected precision.

\subsection{Absolute RVs}

In addition to measuring relative RVs for the stars in our sample, we also measured absolute RVs for these stars using a somewhat modified version of our technique. Previous groups have made use of CPS spectra for measuring absolute RVs: for example, Chubak et al. (2012) analyzed over 29,000 spectra of 2046 F-, G-, K-, and M-type stars (see also Nidever et al. 2002). In this work, we analyzed an additional $~ 3000$ spectra of 213 more massive A- and B-type stars that were not included in Chubak et al. (2012).

We measured the absolute RV for each star in our sample using the same algorithms described in Section 2. However, instead of using a spectrum of the star itself as a template, we performed the fit using a PHOENIX model stellar spectrum of a hot A- or B-type star, using model:

$$
\begin{aligned}
\mathcal{I}_{m}(i, n)= & F(i, n) \cdot S_{m}\left[\lambda_{\text {extrap }}(i, n)\left(1+\frac{V_{\text {Dop }}}{c}\right)\right] \\
& * G\left[V_{\text {rot }} \sin I, R\right]
\end{aligned}
$$

which is similar in form to Equation (4): $F(i, n)$ is the continuum level of the datum spectrum, $S_{m}$ is the unperturbed flux level of the PHOENIX spectrum model, $I$ is the inclination of the stellar spin axis with respect to the line of sight, $V_{\text {rot }}$ is the equatorial stellar rotation velocity, the " $*$ " symbol denotes a convolution, and $G\left[V_{\text {rot }} \sin I, R\right]$ is the broadening kernel, defined by:

$$
\begin{aligned}
& G\left[V_{\text {rot }} \sin I, R\right]=\left(\frac{2(1-\epsilon)}{\pi(1-\epsilon / 2)}\right)\left(1-\left(\Delta \lambda / \Delta \lambda_{L}\right)^{2}\right)^{0.5} \\
& \quad+\left(\frac{\pi \epsilon}{2 \pi(1-\epsilon / 3)}\right)\left(1-\left(\Delta \lambda / \Delta \lambda_{L}\right)^{2}\right)
\end{aligned}
$$

when $R$ is resolution, in units of $\delta \lambda$ per pixel; $\Delta \lambda / \Delta \lambda_{L}$ is a unitless argument, bounded by -1 and 1 describing the position on the star at which the kernel is to be evaluated; $\epsilon$ is the limbdarkening coefficient (taken to be $\epsilon=0.6$ in our analysis, Gray 1976).

The PHOENIX atmospheric models have been developed over the last $15 \mathrm{yr}$ for modeling the spectra of wide range of stellar masses and spectral types (Hauschildt et al. 1999; Jack et al. 2009). The model spectra used in this analysis have metallicities as given in Asplund et al. (2009). The models were generated from effective stellar temperatures as available in the literature for each individual star, ignoring metallicity and surface gravity variations: our fits do not derive stellar qualities beyond the Doppler shift, and thus all we require from a model is the best possible template for deep, broad emission lines. Although using the PHOENIX spectrum as the model requires significantly more exploration through parameterspace to find the Doppler shift, continuum shape, and linebroadening parameters, the result is a description of the absolute motion of the star. We applied a barycentric correction and a slight correction to match the zero-point set by IAU standard stars (e.g., Nidever et al. 2002; Chubak et al. 2012; Collins et al. 2014). We do not correct for the gravitational redshift due to either the host star or our own Sun, as those effects would be on the order of a few $\mathrm{m} \mathrm{s}^{-1}$, which is well below our target precision (Wright \& Eastman 2014). 

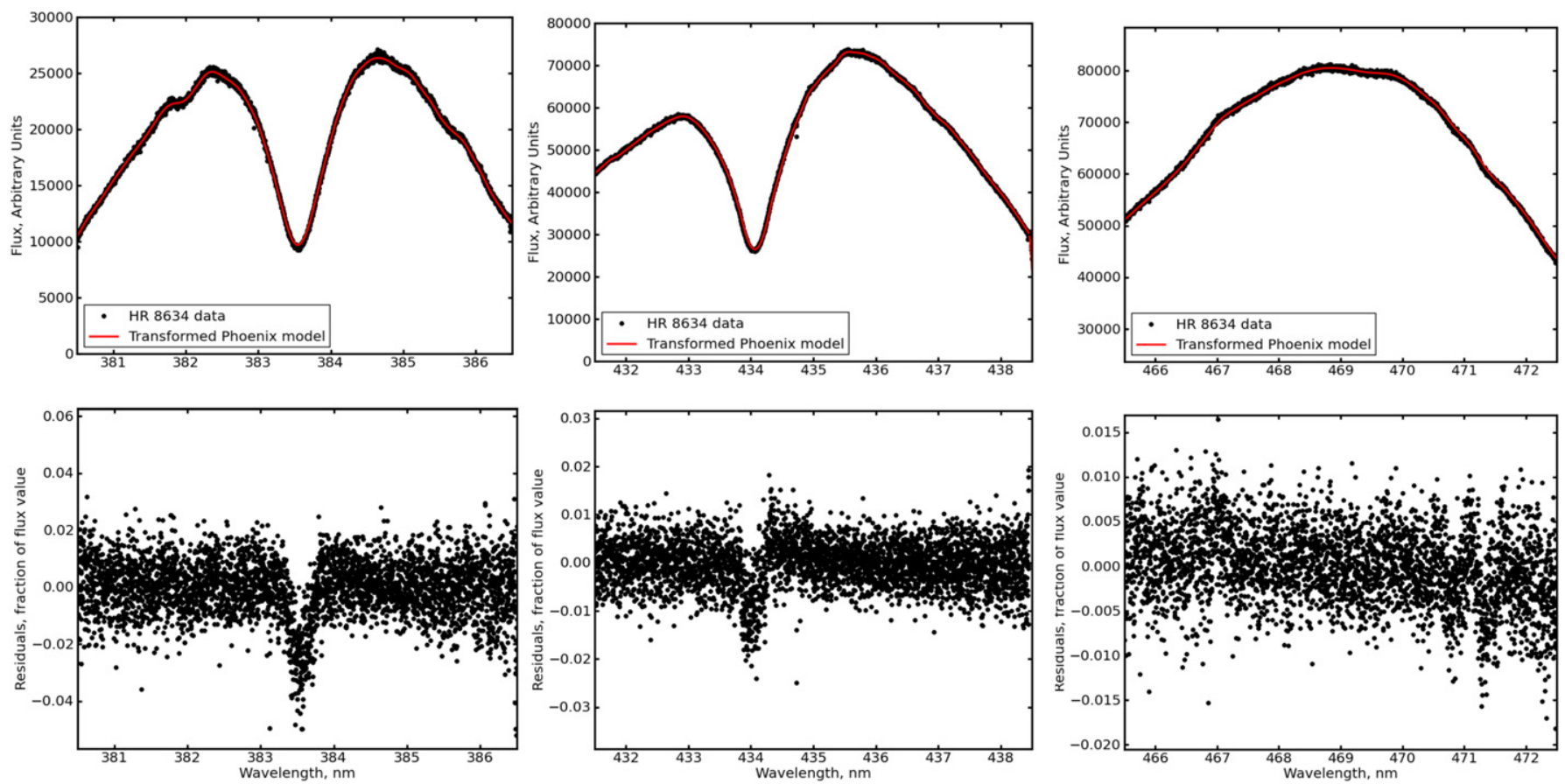

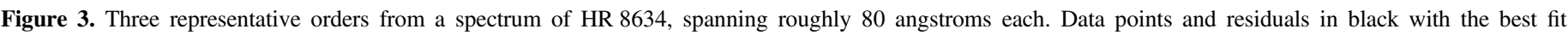

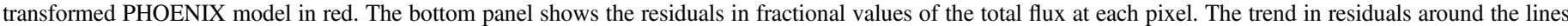

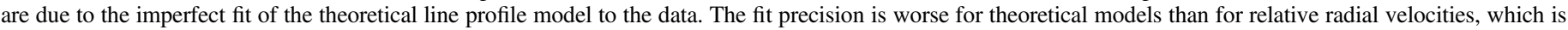
part of why the absolute radial velocity precision is worse than the relative radial velocity precision.

Although our method is not optimized to find $V \sin I$, this quantity is a byproduct of our fits and so we present derived values of $V \sin I$ and its error, $\sigma_{V} \sin I$, for each star in our sample in Table 2.5, assuming zero stellar turbulence.

A final fit to a model and residuals are shown in Figure 3. As evident in the figure, there is some discrepancy between the PHOENIX model atmosphere and the observations. Much of the mismatch is in the line core. However, this is not a major concern given that the Doppler content is contained in the line wings.

\section{RESULTS}

We applied our RV measurement technique to observations of our sample of 213 hot stars. To narrow this sample to contain only those potentially in binary stars, we selected a subsample containing stars that had been observed more than seven times with more than three epochs of observations and additionally showed a large $\left(>3 \sigma\right.$, when $\sigma \approx 1 \mathrm{~km} \mathrm{~s}^{-1}$ is our method precision) amount of scatter in their relative RVs. Here, epoch refers to the night upon which the observation was taken (such that if many spectra were taken in a single night, it would only be one epoch of observations). This sub-sample contains 13 stars that fit these criteria. Of these, one (HR 1178) was excluded from the sample due to being a blended binary as identified in literature, two were excluded due to a significant number of their observations being taken during twilight, leaving 10 stars for further analysis. Of these, three are in detectable multiple systems. We describe our measurements for each of these stars individually in Section 3.3. We also extracted RVs for all 213 stars and report absolute RVs in Table 5 .

Many observations were taken sequentially within the same night, often in clusters of three observations within $\sim 5$ minutes.
To calculate an appropriate bin size for these points, we found the upper limit of semi-amplitude that could occur due to a single-lined companion at the smallest orbital radii possible. We found it was possible to bin data in 4 minute intervals for A-type stars and 15 minute intervals for B-type stars without risk of missing extremely short-period binaries.

\subsection{Measured RV Precision}

We used the RV measurements of the stars with extended time series to both estimate the RV precision of our method, and set limits on any RV trends. To do this, we fit the measured $\mathrm{RV}$ s to a linear model, including a term for RV jitter, using a Markov Chain Monte Carlo (MCMC) algorithm with an affine invariant ensemble sampler (adapted for IDL from the algorithm of Goodman \& Weare 2010; Foreman-Mackey et al. 2013). Our best-fit RV trends and jitter are reported in Table 1.

We find that typically, for stars without known binary companions, the best-fit jitter is between 0.5 and $2 \mathrm{~km} \mathrm{~s}^{-1}$, which we take as the typical precision of our technique. Our data for some stars without known binary companions are consistent with higher values of jitter because the stars have fewer measurements to constrain jitter. Finally, we detect two significant RV trends, which we discuss further in Section 3.3.

\subsection{Absolute RVs}

We report our measurements of absolute RVs for all 213 stars in our sample in Table 5. Table 5 lists the average absolute RV over all observations of each star in our sample, as well as the number of observations and the time baseline of all observations. We report the time baseline of the observations to help avoid contamination with spectroscopic binaries-With a long enough baseline of observations it is possible to separate 
Table 1

Best-fit Linear Trends and RV Jitter

\begin{tabular}{lccc}
\hline \hline Star & Trend $\left(\mathrm{km} \mathrm{s}^{-1} \mathrm{yr}^{-1}\right)$ & Significance $(\sigma)$ & Jitter $\left(\mathrm{km} \mathrm{s}^{-1}\right)$ \\
\hline HR 1679 & $-0.16 \pm 4.38$ & 0.04 & $7.3 \pm 4.3$ \\
HR 2845 & $0.33 \pm 0.43$ & 0.77 & $2.0 \pm 0.6$ \\
HR 3799 & $-0.04 \pm 2.09$ & 0.02 & $2.3 \pm 1.0$ \\
HR 4468 & $-0.33 \pm 0.23$ & 1.41 & $0.9 \pm 0.2$ \\
HR 5511 & $0.09 \pm 0.16$ & 0.54 & $1.5 \pm 0.3$ \\
HR 5849 & $0.10 \pm 0.35$ & 0.29 & $1.0 \pm 0.3$ \\
HR 5867 & $0.96 \pm 0.21$ & 4.52 & $0.5 \pm 0.2$ \\
HR 7708 & $-0.36 \pm 2.59$ & 0.14 & $13.4 \pm 2.7$ \\
HR 8028 & $-1.58 \pm 0.29$ & 5.51 & $1.3 \pm 0.3$ \\
\hline
\end{tabular}

Note. Significance $\sigma$ refers to the magnitude of the trend divided by its uncertainty. Stars with too few $(<7)$ data points are excluded from this table. The best-fit jitter values indicate our precision is typically $1-2 \mathrm{~km} \mathrm{~s}^{-1}$. Note that HR 1679 and HR 7708 have higher levels of scatter than is typical of this technique, possibly indicating close binary companions. HR 3067, discussed in Section 3.3.1 is excluded from this table.

the overall motion of the target from the periodic motion due to companions, and we indeed see some stars with obvious Keplerian motion. However, without a sufficiently long baseline, it is unclear whether an observed RV is due to the star's absolute motion or if it contains an instantaneous snapshot of a star's motion due to the effect of a companion. Only a subset of the A- and B-type calibrator stars were observed a sufficient number of times over a long enough time baseline to make an informed statement on their RVs over time. For this reason, the $T_{\text {baseline }}$ in days is included in Table 5 , to provide a context for each absolute RV measurement.

Both Chubak et al. (2012) and Nidever et al. (2002) were able to calculate absolute RVs for F-, G-, K-, and M-type stars to a precision of roughly $0.1 \mathrm{~km} \mathrm{~s}^{-1}$. We find that our method yields a median precision of $1.5 \mathrm{~km} \mathrm{~s}^{-1}$ (which can be further delineated into a best-case scenario precision as good as 0.5 $\mathrm{km} \mathrm{s}^{-1}$ for low-mass, A-type stars, and a worst-case scenario of $2 \mathrm{~km} \mathrm{~s}^{-1}$ for massive, rapidly rotating B-type stars). In Figure 4, we plot a schematic comparison between our derived values and the compilation presented in Gontcharov (2006). We adopt $1.5 \mathrm{~km} \mathrm{~s}^{-1}$ as the typical uncertainty for our absolute measurements, which is somewhat higher than our errors for relative RVs, due to discrepancies between the PHOENIX models and the observed spectra.

\subsection{Binary Systems}

In our sample of 213 stars, each star has an average of 13 spectra covering 3 epochs. With such sparse temporal sampling, it is difficult to find true periods and fit orbits. Lomb-Scargle analysis of the RV time series for each target often finds spurious short-period signals, due to aliasing, which is particularly troublesome for sparsely sampled targets (Dawson \& Fabrycky 2010). For this reason, we rely on the scatter of the RVs compared to the measurement uncertainties as a simple indicator of the potential presence of a companion, and then consider the data in the context of what is already present in the literature about these sources. Using literature periods as starting points, we are able to confirm and, in some cases, refine what has been reported about the binarity of these massive, bright stars.

Many of the targets in our sample are previously studied binaries (Chini et al. 2012). We observe one blended double- lined binary, HR 1178 (Abt et al. 1965; Zwahlen et al. 2004), which we exclude from our sample because our modeling technique does not account for multiple lines in the spectra. Our method is optimized to find single-lined binaries, and is not presently capable of dealing with double-lined binaries.

We additionally exclude sources with high scatter but insufficient phase coverage, that is, observations at fewer than seven epochs. Out of the 10 stars with at least 7 observations, we identify 2 previously unknown binary systems. We additionally detect the stellar companion to HR 3067, previously found using methods other than RVs. We summarize literature and our observations for each of these systems.

\subsection{1. $H R 3067$}

HR 3067 is a bright star with spectral type A3, and was observed by CPS 23 times over a time baseline of $5 \mathrm{yr}$. Astrometric observations from Hipparcos identified a companion with an orbital period of $1.59 \mathrm{yr}$, and fit the data with a zero-eccentricity orbital model. To our knowledge, no RV confirmation of this companion or measurement of the eccentricity exists in the literature. In our data, we indeed detect significant RV variations (a scatter roughly 10 times the measured scatter of the method; see Table 2), and searched for periodic signals with a Lomb-Scargle analysis. The periodogram analysis of the RV time series of HR 3067 finds several potential short-period peaks; however, due to poor sampling and aliasing (e.g., Dawson \& Fabrycky 2010), we cannot uniquely determine the true period.

We fit a Keplerian model to HR 3067's RV time series. We adopted the astrometrically derived period as a starting point for the fit, and fit the RV time series to a Keplerian using the IDL program rvlin (Wright \& Howard 2009). The result is plotted in Figure 5. To determine errors on these parameters, we used a bootstrap Monte Carlo method, as used in Johnson et al. (2007). This is done by subtracting a model generated from the best-fit Keplerian from the measured RVs, then computing the residuals between the two. The residuals are then randomly reassigned to data points, and rvlin used again to fit a new best-fit Keplerian. The mean and standard deviation of a distribution composed of 1000 such trials was adopted as the system parameters and uncertainties.

We estimated HR 3067's mass using the online Padova model interpolator, ${ }^{9}$ which uses the technique of da Silva et al. (2006) to calculate masses based on photometry, parallaxes and spectroscopic parameters. Assuming HR 3067's observed spectral type of A3, a temperature of roughly $8750 \mathrm{~K}$ and solar metallicity, we estimate that it has a mass of roughly $2.2 M_{\odot}$. Given the primary's mass of $2.2 M_{\odot}$ and the period derived from our RVs, the binary mass function of $0.15 M_{\odot}$ translates to a minimum mass for the secondary component of about $1 M_{\odot}$. Additionally, hints of the spectral lines of the secondary component are visible in the HIRES spectra. Follow-up observations and analysis are ongoing, and the result of further analysis will be presented in a future work.

We show our phase folded RV measurements along with our best-fit model in Figure 5. The best-fit orbital parameters are provided in Table 3.

\footnotetext{
http://stev.oapd.inaf.it/cgi-bin/param
} 

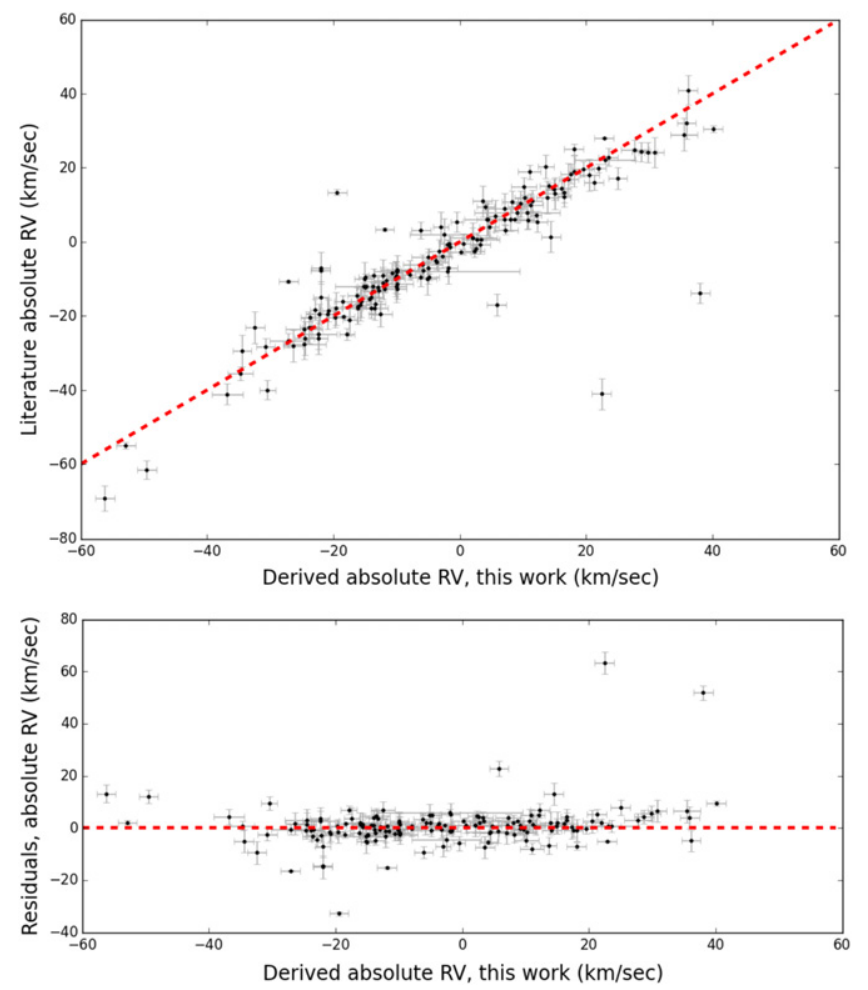

Figure 4. Top panel: a plot of the absolute radial velocity values derived in this work against literature values (drawn from Gontcharov 2006). Bottom panel: residuals between our values and literature values. The rms error is $8.63 \mathrm{~km} \mathrm{~s}^{-1}$

Table 2

Radial Velocity Time Series for HR 3067

\begin{tabular}{lrc}
\hline \hline Date $(\mathrm{JD})$ & Relative RV & Uncertainty \\
\hline 2454808.016881 & -7.99011456 & 0.906307 \\
2454963.801250 & 0.84506144 & 0.851385 \\
2454983.734892 & 2.89520244 & 0.730252 \\
2455255.838258 & 7.40614944 & 1.730396 \\
2455311.718924 & -6.35259256 & 0.912301 \\
2455342.728611 & -14.29464556 & 1.012084 \\
2455343.731059 & -11.11869856 & 0.923183 \\
2455344.732477 & -13.89147756 & 0.842331 \\
2455671.717002 & 4.87441244 & 1.175328 \\
2455672.718090 & 4.98378644 & 1.273581 \\
2455673.721030 & 4.81967244 & 1.293804 \\
2455697.725914 & 4.73993644 & 1.377920 \\
2455698.726088 & 5.18161544 & 1.201775 \\
2455699.725868 & 5.49505144 & 1.187538 \\
2455700.725764 & 5.18047444 & 1.042351 \\
2455702.741458 & 7.22616644 & 0.986378 \\
2456907.153611 & -5.3110145 & 1.071243 \\
2456908.154329 & -5.6374542 & 1.141087 \\
2456909.154225 & -5.9577133 & 1.184617 \\
2456910.146331 & -4.2697303 & 0.918832 \\
2456911.145278 & -4.5781529 & 1.943838 \\
2456912.153484 & -4.8835522 & 1.307474 \\
2456913.153634 & -3.1805621 & 1.234314 \\
\hline
\end{tabular}

\subsection{2. $\operatorname{HR} 5867$}

HR 5867 is an A3-type star that has been studied in the past and found to have many different possible companions, of varying separations. van de Kamp \& Vyssotsky (1929) identified HR 5867 as a quadruple system, the components of

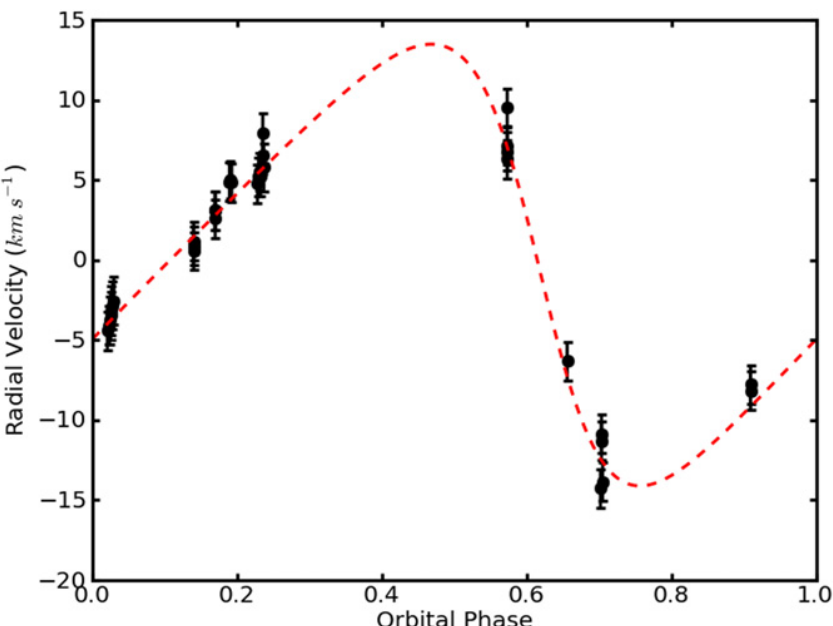

Figure 5. The phase-folded radial velocity time series of HR 3067, an A-type star with a predicted companion at an orbital period of $1.5 \mathrm{yr}$ (Malkov et al. 2012). The RVs show an orbit with a longer period than astrometry predicted and significant eccentricity.

Table 3

Best-fit Orbital Parameters for HR 3067

\begin{tabular}{lcc}
\hline \hline Orbital Parameter & Value & Uncertainty \\
\hline$p$ & 674.64 days & 7.35 days \\
$e$ & 0.38 & 0.05 \\
$\omega$ & $93: 91$ & $3: 12$ \\
$k$ & $13.80 \mathrm{~km} \mathrm{~s}^{-1}$ & $0.79 \mathrm{~km} \mathrm{~s}^{-1}$
\end{tabular}

which were separated by $30^{\prime \prime}$ from the primary. Shaya \& Olling (2011) reported a very wide companion to HR 5867. De Rosa et al. (2014) identified a further companion at 1643.04 arcsec.

We find a linear trend in our new RVs, suggesting the presence of a close-in, previously unstudied companion. Our new RVs span a baseline of more than 1000 days, but do not catch a turn-over in the RV curve. As shown in Figure 6, an MCMC fit to a linear model finds a significant slope, indicating long-term motion in the star. This companion has not been previously identified. Since we have a measure of the RV trend as well as an astrometric measure of the separation between the primary and visible companions, we can determine the companion mass using Knutson et al. (2014):

$$
M_{\text {comp }} / M_{\odot}=5.34 \times 10^{-6}\left(\frac{d}{\mathrm{pc}} \frac{p}{\operatorname{arcsec}}\right)^{2}\left|\frac{d(\mathrm{RV})}{d t}\right| \Phi
$$

when $d$ is distance to the system, $p$ is separation in arcseconds between the primary and companion, $\frac{d(\mathrm{RV})}{d t}$ is the RV trend, and $\Phi$ is a function of the inclination angle, eccentricity, longitude of periastron, and phase in orbit, which assumes a minimum value of $\sqrt{27} / 2$ (Torres 1999; Liu et al. 2002; Knutson et al. 2014). Using Equation (7), van Leeuwen (2007)'s measured parallax of 21.03 mas, and the separations of the previously measured companions (van de Kamp \& Vyssotsky 1929; Shaya \& Olling 2011; De Rosa et al. 2014), and our new value of $\frac{d(\mathrm{RV})}{d t}=0.96 \mathrm{~km} \mathrm{~s}^{-1} \mathrm{yr}^{-1}$, we find that if the $\mathrm{RV}$ trend we observe was caused by one of the known visual binary companions to HR 5867, the companion would have a minimum mass of $2 \times 10^{4} M_{\odot}$. This result is unphysical, so we 

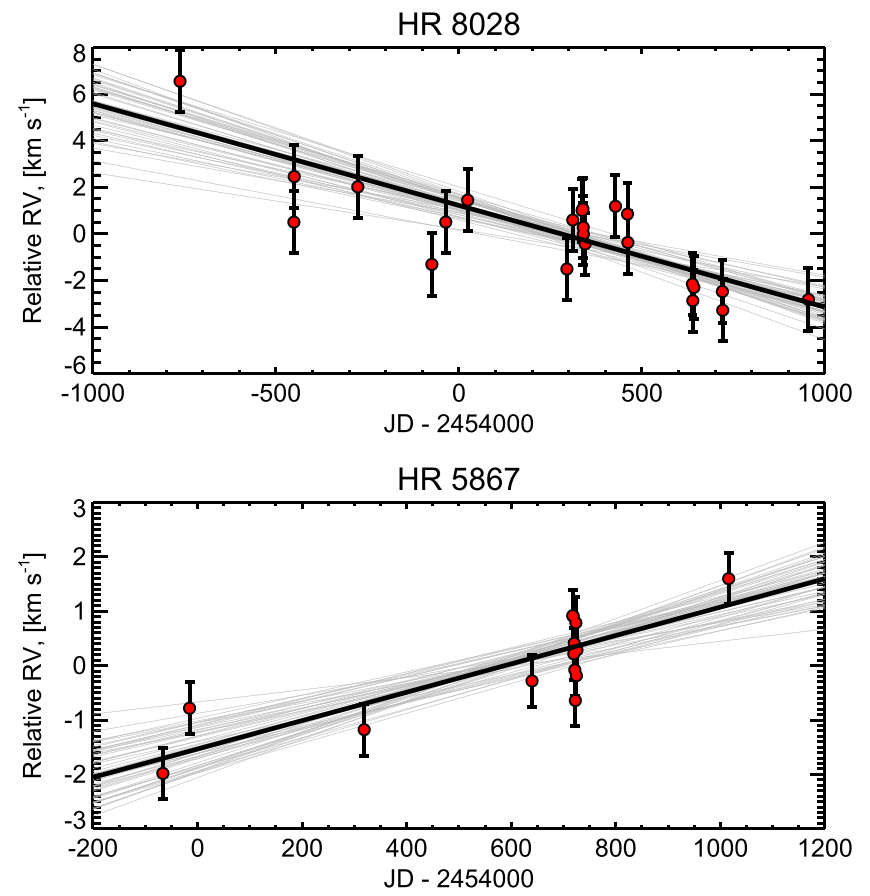

Figure 6. Top: a linear fit to the radial velocity points for HR 5867 excludes a zero slope (no trend) to $4.5 \sigma$. Gray lines show random draws from the MCMC posterior. This indicates the presence of a companion forcing the trend, inducing an amplitude variation of $0.96 \mathrm{~km} \mathrm{~s}^{-1} \mathrm{yr}^{-1}$. Bottom: Same as top, but for HR 8028. We attribute this trend to a companion detected in speckle imaging of this star. The best-fit trend is $-1.58 \mathrm{~km} \mathrm{~s}^{-1} \mathrm{yr}^{-1}$, and is significant at the $5.5 \sigma$ level.

conclude that the companion inciting the RV trend that we see must be an undiscovered companion.

Estimating HR 5867 as a $2.2 M_{\odot}$ star using the Padova interpolator described above, we can estimate the mass of the companion using (Wright et al. 2007):

$$
\frac{m^{3} \sin ^{3} i}{\left(m+M_{*}\right)^{2}}=\frac{P K^{3}\left(1-e^{2}\right)^{3 / 2}}{2 \pi G}
$$

where $m$ is the mass of the companion, $M_{*}$ is the mass of the primary, $P$ is the period of the orbit, $K$ is the amplitude of the $\mathrm{RV}$ signal, and $e$ is the eccentricity. Using the minimum amplitude for $\operatorname{HR} 5867\left(K \geqslant 3.15 \mathrm{~km} \mathrm{~s}^{-1}\right)$, the minimum period $(P \geqslant 6.3 \mathrm{yr})$, and assuming that $e=0$ and $M_{*}=2.2 M_{\odot}$ and $i=90^{\circ}$, we find that the companion must have a minimum mass of $m \geqslant 0.37 M_{\odot}$. This could be further constrained with additional RV measurements, particularly if the new data cover an inflection point.

\subsection{3. $H R 8028$}

HR 8028 is an A1-type, main-sequence star with a speckle companion roughly $0.1^{\prime \prime}$ away (Horch et al. 2012). The astrometric separation has been measured several times: these values are presented in Table 4, along with the instantaneous separations derived using HR 8028's parallax, which is 8.71 mas \pm 0.34 mas (van Leeuwen 2007), corresponding to a distance of $114 \pm 4$ parsecs .

As shown in Figure 6, an MCMC fit to a linear model finds a significant slope, indicating long-term motion in the star. The companion inciting this motion must have a minimum period of $10 \mathrm{yr}$, consistent with the orbital separations found via astrometry by Mason et al. (1999), Horch et al. (2008), and Horch et al. (2012). The $\frac{d v}{d t}=1.58 \mathrm{~km} \mathrm{~s}^{-1} \mathrm{yr}^{-1}$ and separations in Table 4 allows us to calculate a minimum dynamical mass for the companion based on the local RV slope, assuming a mass of $2.3 M_{\odot}$ (from the Padova interpolator) for the A1 primary. Using Equation (7) and the separations summarized in Table 4, we find the mass of the companion to be a minimum 2 $M_{\odot}$, which would make the companion of comparable size to the primary. This is unlikely, considering the magnitude difference between the primary and secondary evident in speckle imaging (2.1 magnitudes, Mason et al. 1999). The speckle companion seems to be moving relative to the primary (roughly 0.3 arcsec in $20 \mathrm{yr}$ ) at a rate comparable to the proper motion of the primary $(0.5 \operatorname{arcsec}$ in $20 \mathrm{yr})$. It is possible that the speckle companion is a background star, and the companion we detect in RVs is different. Using Equation (8) with lower bounds of $8 \mathrm{yr}$ for the period (due to unconstrained eccentricity, we cannot exclude a $8 \mathrm{yr}$ period for an eccentric orbit) and $5 \mathrm{~km} \mathrm{~s}^{-1}$ for the $\mathrm{RV}$ amplitude yields a minimum mass companion of $m \geqslant 0.7 M_{\odot}$. The period and amplitude both have only lower limits due to the lack of turn-over in the RV curve. Further refinement of the period of HR 8028's companion (and thus lower bounds on the its mass) will be possible once a turnover is measured in the RV measurements.

\section{DISCUSSION}

In this work, we present data on some of the closest, brightest stars in the sky. Even though these stars have been studied for over a century, it is still possible to make discoveries by using existing data in new ways.

Some of our absolute RV measurements are new and have not yet been presented in literature, while many others serve as an update to previous literature values. These new measurements will serve as additional reference measurements for programs studying the kinematics of bulk stellar flow. The updated absolute RVs can also be combined with with longterm measurements of these stars to extend the time baseline of RV monitoring of these sources. Moreover, additional absolute RV measurements will help the Hundred-Thousand-ProperMotion survey. de Bruijne \& Eilers (2012) found that many sources, including some in our sample, need additional RV measurements to be useful to the Hundred-Thousand-ProperMotion survey.

Calibrator spectra are an underutilized resource with the potential to do new science. Our method of fitting RVs for massive stars could be applied to spectrographs other than HIRES, allowing for more RV measurements of various types of rapidly rotating stars. A compilation of calibration spectra from other spectrographs could significantly increase the size of our dataset and help the sampling issues that prevented us from identifying more spectroscopic binaries. Infrared spectrographs in particular could substantially increase the number of calibration spectra, because taking spectra of rapidly rotating hot stars to calibrate spectral features from Earth's atmosphere is common practice among infrared astronomers (e.g., Muirhead et al. 2013).

Additionally, though this method was developed for use on A- and B-type stars, it can be used for any target with broadened spectral features. A modified version of this method was used in Muirhead et al. (2013) to find RVs for a rotating M3-dwarf with a $V_{\text {rot }} \sin i=19.67 \pm 0.52 \mathrm{~km} \mathrm{~s}^{-1}$. This 
Table 4

Astrometric Measurements for HR 8028

\begin{tabular}{|c|c|c|c|c|}
\hline Date & $\rho$, Ang. Sep $(\operatorname{arcsec})$ & Position Angle $\left({ }^{\circ}\right)$ & Separation(AU) & Reference \\
\hline 1989.7114 & 0.262 & 89.7114 & 29.9 & Mason et al. (1999) \\
\hline 2003.5383 & 0.084 & 204.6 & 9.6 & Horch et al. (2008) \\
\hline 2003.5384 & 0.0849 & 202.1 & 9.6 & Horch et al. (2008) \\
\hline 2008.4722 & 0.113 & 169.8 & 12.9 & Horch et al. (2012) \\
\hline 2009.4498 & 0.140 & 177.5 & 16 & Horch et al. (2012) \\
\hline 2009.4498 & 0.144 & 175.0 & 16.4 & Horch et al. (2012) \\
\hline 2009.4578 & 0.137 & 176.2 & 15.6 & Horch et al. (2012) \\
\hline
\end{tabular}

Table 5

Summary of all Observations

\begin{tabular}{|c|c|c|c|c|c|c|c|}
\hline Star & Abs. $\mathrm{RV}\left(\mathrm{km} \mathrm{s}^{-1}\right)$ & $\sigma_{R V}\left(\mathrm{~km} \mathrm{~s}^{-1}\right)$ & $V \sin (I)\left(\mathrm{km} \mathrm{s}^{-1}\right)$ & $\sigma_{V \sin (I)}\left(\mathrm{km} \mathrm{s}^{-1}\right)$ & $N_{\text {obs }}$ & $T_{\text {baseline }}$ (days) & Notes \\
\hline$\overline{\text { HR10 }}$ & -10.92 & 1.5 & 252 & 17 & 6 & 462.677778 & $\mathrm{c}$ \\
\hline HR1002 & -10.06 & 1.00 & 132 & 6 & 13 & 1098.160023 & $\ldots$ \\
\hline HR1062 & 14.11 & 1.5 & 120 & 13 & 7 & 175.637489 & $\mathrm{c}$ \\
\hline HR1087 & -1.67 & 1.5 & 190 & 14 & 10 & 0.018322 & $\mathrm{~d}$ \\
\hline HR1239 & 16.52 & 0.40 & 38 & 11 & 16 & 1834.113172 & $\ldots$ \\
\hline HR1260 & 5.82 & 1.5 & 168 & 23 & 6 & 0.089051 & $\mathrm{c}, \mathrm{d}$ \\
\hline HR1261 & 7.11 & 0.91 & 251 & 15 & 12 & 392.0197 & $\ldots$ \\
\hline HR1273 & 4.07 & 1.39 & 55 & 9 & 10 & 767.998484 & $\ldots$ \\
\hline HR128 & -21.99 & 1.17 & 194 & 13 & 9 & 434.995069 & $\ldots$ \\
\hline HR1289 & -22.01 & 1.5 & 265 & 10 & 3 & 0.001412 & $\mathrm{c}, \mathrm{d}$ \\
\hline HR15 & -6.35 & 1.5 & 95 & 40 & 3 & 1236.918252 & $\mathrm{~d}$ \\
\hline HR1500 & 12.24 & 1.55 & 249 & 23 & 12 & 69.792176 & $\ldots$ \\
\hline HR1544 & 28.70 & 1.5 & 228 & 19 & 7 & 1097.996562 & $\mathrm{~d}$ \\
\hline HR1567 & 30.78 & 1.5 & 77 & 27 & 3 & 0.087615 & $\mathrm{c}, \mathrm{d}$ \\
\hline HR1574 & 10.12 & 1.97 & 155 & 14 & 15 & 86.672651 & \\
\hline HR1621 & 29.78 & 1.5 & 312 & 26 & 6 & 447.719641 & $\mathrm{c}$ \\
\hline HR1641 & 12.09 & 0.85 & 115 & 15 & 35 & 2361.619584 & \\
\hline HR1679 & -2.51 & 5.43 & 327 & 24 & 37 & 978.285498 & $\mathrm{a}$ \\
\hline HR1786 & -10.09 & 1.5 & 190 & 19 & 6 & 0.002546 & $\mathrm{c}, \mathrm{d}$ \\
\hline HR1789 & 19.98 & 1.5 & 287 & 24 & 6 & 28.939711 & $\mathrm{c}, \mathrm{d}$ \\
\hline HR179 & -12.58 & 1.75 & 134 & 8 & 11 & 1236.393646 & $\cdots$ \\
\hline HR1806 & 17.51 & 1.5 & 202 & 41 & 3 & 0.001783 & $\mathrm{c}, \mathrm{d}$ \\
\hline HR1858 & 36.12 & 1.5 & 199 & 26 & 3 & 0.001342 & $\mathrm{c}, \mathrm{d}$ \\
\hline HR1873 & 5.65 & 1.5 & 231 & 29 & 3 & 0.001632 & $\mathrm{c}, \mathrm{d}$ \\
\hline HR193 & -14.93 & 1.5 & 221 & 16 & 7 & 688.042025 & $\mathrm{c}$ \\
\hline HR2155 & 35.89 & 1.5 & 234 & 15 & 3 & 0.00169 & $\mathrm{c}, \mathrm{d}$ \\
\hline HR2198 & 19.54 & 1.72 & 248 & 8 & 14 & 507.819283 & $\ldots$ \\
\hline HR2209 & -13.26 & 1.5 & 245 & 31 & 3 & 0.001412 & $\mathrm{c}, \mathrm{d}$ \\
\hline HR223 & 7.01 & 2.97 & 115 & 13 & 21 & 765.087974 & $\ldots$ \\
\hline HR2231 & 22.83 & 1.5 & 244 & 22 & 5 & 1.993738 & $\mathrm{c}, \mathrm{d}$ \\
\hline HR2297 & 35.44 & 2.13 & 174 & 12 & 6 & 95.867338 & c \\
\hline HR2343 & 41.73 & 0.92 & 205 & 11 & 23 & 264.288344 & $\ldots$ \\
\hline HR2356 & 24.98 & 1.5 & 369 & 14 & 4 & 0.002175 & $\mathrm{c}, \mathrm{d}$ \\
\hline HR2370 & 23.50 & 1.5 & 379 & 32 & 4 & 0.002465 & $\mathrm{c}, \mathrm{d}$ \\
\hline HR2490 & 7.22 & 1.92 & 121 & 25 & 9 & 285.170532 & $\ldots$ \\
\hline HR2532 & 4.25 & 1.5 & 275 & 12 & 3 & 0.001679 & $\mathrm{c}, \mathrm{d}$ \\
\hline HR2568 & -36.83 & 2.45 & 248 & 16 & 9 & 469.783901 & $\ldots$ \\
\hline HR2585 & -13.71 & 1.50 & 217 & 15 & 21 & 960.236042 & $\ldots$ \\
\hline HR26 & -19.44 & 1.5 & 205 & 21 & 6 & 913.119676 & $\mathrm{c}$ \\
\hline HR2648 & 27.72 & 2.20 & 338 & 19 & 16 & 356.01978 & $\ldots$ \\
\hline HR2670 & 22.85 & 1.5 & 261 & 19 & 3 & 0.001759 & $\mathrm{c}, \mathrm{d}$ \\
\hline HR2763 & -9.86 & 2.75 & 148 & 6 & 12 & 156.812257 & $\ldots$ \\
\hline HR2783 & 7.48 & 2.63 & 286 & 20 & 15 & 117.72103 & $\ldots$ \\
\hline HR2845 & 17.30 & 3.01 & 248 & 13 & 50 & 1739.255972 & $\mathrm{a}$ \\
\hline HR2944 & 51.47 & 1.5 & 126 & 18 & 4 & 0.002072 & $\mathrm{c}, \mathrm{d}$ \\
\hline HR2946 & 9.16 & 1.5 & 150 & 8 & 4 & 788.915614 & $\mathrm{c}$ \\
\hline HR3034 & 35.73 & 1.5 & 412 & 135 & 7 & 303.167615 & $\mathrm{c}$ \\
\hline HR3067 & 9.07 & 6.80 & 158 & 23 & 23 & 895.712859 & $\mathrm{a}$ \\
\hline HR311 & -12.38 & 1.5 & 251 & 12 & 7 & 355.911065 & $\mathrm{c}$ \\
\hline HR3131 & -13.81 & 1.5 & 255 & 15 & 3 & 0.001216 & $\mathrm{c}, \mathrm{d}$ \\
\hline
\end{tabular}


Table 5

(Continued)

\begin{tabular}{|c|c|c|c|c|c|c|c|}
\hline Star & Abs. $\mathrm{RV}\left(\mathrm{km} \mathrm{s}^{-1}\right)$ & $\sigma_{R V}\left(\mathrm{~km} \mathrm{~s}^{-1}\right)$ & $V \sin (I)\left(\mathrm{km} \mathrm{s}^{-1}\right)$ & $\sigma_{V \sin (I)}\left(\mathrm{km} \mathrm{s}^{-1}\right)$ & $N_{\text {obs }}$ & $T_{\text {baseline }}$ (days) & Notes \\
\hline HR3134 & -10.00 & 1.5 & 189 & 22 & 6 & 0.034942 & $\mathrm{c}, \mathrm{d}$ \\
\hline HR3173 & 3.56 & 1.5 & 173 & 24 & 3 & 0.001273 & $\mathrm{c}, \mathrm{d}$ \\
\hline HR3192 & 22.01 & 1.09 & 130 & 22 & 14 & 94.78103 & $\ldots$ \\
\hline HR3474 & 18.04 & 1.5 & 180 & 18 & 6 & 257.361504 & $\mathrm{c}$ \\
\hline HR3601 & -17.43 & 1.18 & 160 & 11 & 9 & 1094.019051 & $\ldots$ \\
\hline HR3662 & -16.13 & 1.30 & 158 & 17 & 10 & 205.197732 & $\ldots$ \\
\hline HR3665 & -27.14 & 1.5 & 111 & 19 & 3 & 0.001169 & $\mathrm{c}, \mathrm{d}$ \\
\hline HR3690 & 4.65 & 1.5 & 165 & 10 & 6 & 764.881991 & $\mathrm{c}$ \\
\hline HR3799 & 23.03 & 4.76 & 204 & 26 & 35 & 568.760173 & $\mathrm{a}$ \\
\hline HR384 & -16.25 & 1.5 & 363 & 33 & 3 & 0.001551 & $\mathrm{c}, \mathrm{d}$ \\
\hline HR3858 & 16.05 & 1.5 & 286 & 27 & 3 & 0.00125 & $\mathrm{c}, \mathrm{d}$ \\
\hline HR3885 & -5.06 & 1.5 & 320 & 15 & 6 & 0.015972 & $\mathrm{c}, \mathrm{d}$ \\
\hline HR3917 & -13.68 & 1.36 & 144 & 12 & 15 & 1052.038044 & $\ldots$ \\
\hline HR398 & 8.23 & 1.5 & 124 & 58 & 3 & 1627.157998 & $\mathrm{c}$ \\
\hline HR3982 & 10.81 & 1.5 & 307 & 13 & 21 & 654.254503 & \\
\hline HR4116 & 18.07 & 0.96 & 130 & 13 & 25 & 1143.034294 & $\ldots$ \\
\hline HR4123 & 15.02 & 1.38 & 243 & 17 & 15 & 754.982338 & $\ldots$ \\
\hline HR4172 & 16.49 & 1.01 & 261 & 12 & 15 & 668.142639 & $\ldots$ \\
\hline HR419 & 13.63 & 1.24 & 160 & 23 & 12 & 410.984386 & \\
\hline HR4259 & -0.49 & 0.98 & 176 & 24 & 13 & 390.999907 & \\
\hline HR4260 & 1.30 & 0.92 & 219 & 16 & 16 & 521.426806 & \\
\hline HR4317 & 40.07 & 1.5 & 107 & 34 & 3 & 0.002488 & $\mathrm{c}, \mathrm{d}$ \\
\hline HR4388 & -5.19 & 1.5 & 255 & 22 & 6 & 91.775648 & $\mathrm{c}$ \\
\hline HR4422 & -9.86 & 2.46 & 181 & 11 & 39 & 881.752685 & $\ldots$ \\
\hline HR4468 & 2.09 & 3.78 & 204 & 8 & 52 & 1608.756203 & $\mathrm{a}$ \\
\hline HR4515 & 0.56 & 1.13 & 138 & 9 & 21 & 1123.912442 & $\ldots$ \\
\hline HR4787 & -14.89 & 1.27 & 132 & 16 & 12 & 521.674422 & $\ldots$ \\
\hline HR4828 & 3.23 & 1.61 & 139 & 12 & 36 & 1181.780093 & $\ldots$ \\
\hline HR4875 & -13.32 & 1.5 & 173 & 27 & 5 & 385.056157 & $\mathrm{c}$ \\
\hline HR4886 & -15.14 & 1.5 & 239 & 45 & 5 & 54.920266 & $\mathrm{c}$ \\
\hline HR4936 & -32.45 & 1.5 & 216 & 15 & 7 & 316.121968 & $\mathrm{c}$ \\
\hline HR496 & -4.94 & 2.71 & 387 & 58 & 20 & 901.55287 & \\
\hline HR5037 & -1.87 & 1.5 & 237 & 24 & 7 & 56.930243 & $\mathrm{c}$ \\
\hline HR5062 & -15.01 & 1.02 & 231 & 12 & 13 & 1100.929109 & $\ldots$ \\
\hline HR5107 & -12.89 & 1.12 & 236 & 15 & 15 & 1085.97051 & $\ldots$ \\
\hline HR5112 & -22.90 & 0.83 & 148 & 11 & 30 & 683.14287 & $\ldots$ \\
\hline HR5127 & -11.58 & 2.12 & 185 & 32 & 4 & 0.001979 & $\mathrm{c}, \mathrm{d}$ \\
\hline HR5179 & -13.01 & 1.5 & 224 & 21 & 3 & 0.001482 & $\mathrm{c}, \mathrm{d}$ \\
\hline HR5238 & -13.13 & 1.5 & 242 & 34 & 4 & 0.002616 & $\mathrm{c}, \mathrm{d}$ \\
\hline HR5244 & -23.84 & 1.5 & 121 & 18 & 7 & 768.021759 & $\mathrm{c}$ \\
\hline HR545 & -4.57 & 9.28 & 67 & 11 & 19 & 864.65926 & $\mathrm{a}$ \\
\hline HR5478 & 9.05 & 1.36 & 107 & 21 & 17 & 776.813275 & $\ldots$ \\
\hline HR5511 & -5.88 & 3.12 & 262 & 14 & 77 & 2685.67243 & $\mathrm{a}$ \\
\hline HR5517 & 4.39 & 3.73 & 142 & 10 & 10 & 858.685995 & $\mathrm{a}$ \\
\hline HR5685 & -34.76 & 1.95 & 213 & 12 & 15 & 19.981123 & $\ldots$ \\
\hline HR5735 & -2.81 & 1.5 & 193 & 57 & 3 & 0.0011 & $\mathrm{c}, \mathrm{d}$ \\
\hline HR5849 & -15.26 & 4.54 & 182 & 12 & 6 & 1574.728785 & $\mathrm{a}$ \\
\hline HR586 & -27.14 & 2.97 & 306 & 37 & 9 & 1970.238947 & $\ldots$ \\
\hline HR5867 & 3.32 & 3.01 & 211 & 8 & 32 & 1082.189607 & $\mathrm{~b}$ \\
\hline HR5938 & -18.44 & 1.75 & 256 & 49 & 29 & 769.839537 & $\ldots$ \\
\hline HR5949 & -9.88 & 1.64 & 162 & 12 & 24 & 764.902535 & \\
\hline HR6003 & -16.95 & 1.87 & 36 & 22 & 13 & 29.135775 & $\ldots$ \\
\hline HR6013 & -15.78 & 1.5 & 264 & 16 & 6 & 0.002523 & $\mathrm{c}, \mathrm{d}$ \\
\hline HR6036 & -10.17 & 1.5 & 166 & 25 & 6 & 0.002755 & $\mathrm{c}, \mathrm{d}$ \\
\hline HR6051 & -5.77 & 0.99 & 303 & 12 & 12 & 27.025555 & $\ldots$ \\
\hline HR6054 & -10.66 & 1.5 & 113 & 21 & 6 & 0.024664 & $\mathrm{c}, \mathrm{d}$ \\
\hline HR6110 & 2.65 & 1.5 & 226 & 26 & 6 & 24.98375 & $\mathrm{c}, \mathrm{d}$ \\
\hline HR615 & 38.04 & 1.5 & 239 & 28 & 6 & 343.953368 & $\mathrm{c}$ \\
\hline HR6410 & -30.47 & 1.28 & 315 & 22 & 15 & 1184.671933 & $\ldots$ \\
\hline HR6502 & -30.78 & 1.5 & 263 & 36 & 3 & 0.001342 & $\mathrm{c}, \mathrm{d}$ \\
\hline HR6511 & 5.64 & 1.5 & 321 & 23 & 4 & 0.0025 & $\mathrm{c}, \mathrm{d}$ \\
\hline HR6534 & -22.46 & 1.5 & 230 & 29 & 3 & 0.001377 & $\mathrm{c}, \mathrm{d}$ \\
\hline HR6629 & -22.06 & 1.5 & 176 & 16 & 3 & 2645.684549 & $\mathrm{c}$ \\
\hline HR664 & 11.15 & 2.01 & 237 & 14 & 12 & 478.720694 & $\ldots$ \\
\hline
\end{tabular}


Table 5

(Continued)

\begin{tabular}{|c|c|c|c|c|c|c|c|}
\hline Star & Abs. RV $\left(\mathrm{km} \mathrm{s}^{-1}\right)$ & $\sigma_{R V}\left(\mathrm{~km} \mathrm{~s}^{-1}\right)$ & $V \sin (I)\left(\mathrm{km} \mathrm{s}^{-1}\right)$ & $\sigma_{V \sin (I)}\left(\mathrm{km} \mathrm{s}^{-1}\right)$ & $N_{\text {obs }}$ & $T_{\text {baseline }}$ (days) & Notes \\
\hline HR6700 & -13.55 & 2.16 & 155 & 30 & 8 & 57.966077 & \\
\hline HR6723 & 8.06 & 1.5 & 168 & 26 & 6 & 0.981238 & $\mathrm{c}, \mathrm{d}$ \\
\hline HR6747 & 17.23 & 1.5 & 287 & 36 & 6 & 128.712014 & $\mathrm{c}$ \\
\hline HR6779 & -30.01 & 1.22 & 171 & 24 & 86 & 2263.793761 & $\cdots$ \\
\hline HR6789 & -7.95 & 1.5 & 196 & 41 & 3 & 0.001412 & $\mathrm{c}, \mathrm{d}$ \\
\hline HR6826 & -10.09 & 1.5 & 255 & 33 & 5 & 522.625034 & $\mathrm{c}$ \\
\hline HR6827 & -19.56 & 1.03 & 175 & 19 & 36 & 1156.87287 & \\
\hline HR6873 & -14.94 & 1.5 & 234 & 17 & 7 & 258.202708 & $\mathrm{c}$ \\
\hline HR6881 & -12.15 & 1.5 & 203 & 36 & 3 & 0.001354 & $\mathrm{c}, \mathrm{d}$ \\
\hline HR6923 & -29.82 & 1.5 & 215 & 17 & 6 & 0.026435 & $\mathrm{c}, \mathrm{d}$ \\
\hline HR6930 & 22.43 & 1.5 & 166 & 37 & 3 & 0.001238 & $\mathrm{c}, \mathrm{d}$ \\
\hline HR708 & 11.00 & 1.83 & 234 & 21 & 72 & 1385.298357 & \\
\hline HR7096 & -34.50 & 1.5 & 142 & 17 & 3 & 0.001516 & $\mathrm{c}, \mathrm{d}$ \\
\hline HR7142 & -52.88 & 1.5 & 257 & 44 & 3 & 0.001216 & $\mathrm{c}, \mathrm{d}$ \\
\hline HR7202 & -23.68 & 1.5 & 264 & 31 & 3 & 0.001319 & $\mathrm{c}, \mathrm{d}$ \\
\hline HR7235 & -22.36 & 2.88 & 280 & 17 & 19 & 504.788991 & $\cdots$ \\
\hline HR7236 & -7.98 & 0.96 & 76 & 20 & 9 & 539.651655 & $\ldots$ \\
\hline HR7249 & -19.75 & 1.5 & 186 & 42 & 3 & 0.001262 & $\mathrm{c}, \mathrm{d}$ \\
\hline HR7262 & -24.57 & 1.5 & 259 & 19 & 3 & 0.001354 & $\mathrm{c}, \mathrm{d}$ \\
\hline HR7403 & -19.39 & 1.5 & 315 & 44 & 3 & 0.001944 & $\mathrm{c}, \mathrm{d}$ \\
\hline HR7420 & -22.24 & 2.21 & 219 & 23 & 21 & 1096.953044 & $\ldots$ \\
\hline HR7446 & -20.94 & 1.11 & 263 & 36 & 16 & 828.693796 & $\cdots$ \\
\hline HR7457 & -13.98 & 1.92 & 208 & 79 & 74 & 707.014815 & $\cdots$ \\
\hline HR7466 & -16.93 & 2.46 & 171 & 16 & 30 & 706.036806 & $\ldots$ \\
\hline HR7528 & -24.68 & 2.96 & 168 & 20 & 34 & 1160.874757 & $\ldots$ \\
\hline HR7543 & -26.43 & 1.47 & 232 & 21 & 17 & 133.771922 & $\cdots$ \\
\hline HR7565 & -17.91 & 1.23 & 209 & 36 & 9 & 617.270567 & $\ldots$ \\
\hline HR7600 & -56.19 & 1.5 & 278 & 51 & 6 & 377.831123 & $\mathrm{c}$ \\
\hline HR7708 & -1.98 & 11.46 & 349 & 35 & 64 & 1060.992003 & $\mathrm{a}$ \\
\hline HR7724 & -23.48 & 1.5 & 207 & 53 & 6 & 91.049363 & $\mathrm{c}$ \\
\hline HR7740 & -16.13 & 2.53 & 222 & 26 & 12 & 828.785996 & \\
\hline HR7757 & -12.16 & 2.31 & 184 & 14 & 18 & 704.138507 & $\ldots$ \\
\hline HR7803 & -4.82 & 1.5 & 183 & 30 & 4 & 579.418577 & $\mathrm{c}$ \\
\hline HR7890 & -10.16 & 1.5 & 246 & 49 & 3 & 0.00162 & $\mathrm{c}, \mathrm{d}$ \\
\hline HR7906 & -2.90 & 1.60 & 124 & 13 & 31 & 1085.153819 & $\cdots$ \\
\hline HR793 & -3.31 & 1.5 & 162 & 48 & 3 & 0.001308 & $\mathrm{c}, \mathrm{d}$ \\
\hline HR7950 & -14.33 & 0.85 & 110 & 7 & 12 & 80.817974 & \\
\hline HR801 & 9.83 & 5.62 & 68 & 18 & 27 & 1119.029144 & $\mathrm{a}$ \\
\hline HR8028 & -24.61 & 3.35 & 207 & 7 & 51 & 1717.179247 & $\mathrm{~b}$ \\
\hline HR804 & -3.84 & 1.5 & 179 & 30 & 6 & 389.851169 & $\mathrm{c}$ \\
\hline HR8047 & 14.42 & 1.5 & 334 & 28 & 4 & 0.002257 & $\mathrm{c}, \mathrm{d}$ \\
\hline HR8146 & -1.93 & 0.59 & 230 & 24 & 23 & 1107.927408 & $\cdots$ \\
\hline HR8270 & -20.86 & 0.92 & 261 & 29 & 9 & 489.834769 & \\
\hline HR8319 & 2.35 & 1.5 & 172 & 38 & 3 & 0.001273 & $\mathrm{c}, \mathrm{d}$ \\
\hline HR8342 & -1.53 & 0.82 & 207 & 17 & 12 & 413.913171 & $\ldots$ \\
\hline HR835 & -11.92 & 1.5 & 180 & 16 & 2 & 0.000729 & $\mathrm{c}, \mathrm{d}$ \\
\hline HR8373 & 9.61 & 1.5 & 194 & 40 & 3 & 0.001343 & $\mathrm{c}, \mathrm{d}$ \\
\hline HR838 & -3.01 & 1.21 & 205 & 22 & 202 & 2612.853019 & \\
\hline HR8402 & 11.50 & 1.5 & 224 & 25 & 3 & 0.001389 & $\mathrm{c}, \mathrm{d}$ \\
\hline HR8438 & -49.57 & 1.5 & 201 & 19 & 6 & 955.348137 & $\mathrm{c}$ \\
\hline HR8450 & -10.01 & 0.56 & 159 & 13 & 23 & 152.697998 & \\
\hline HR8451 & -6.20 & 1.5 & 191 & 35 & 6 & 0.013518 & $\mathrm{c}, \mathrm{d}$ \\
\hline HR8597 & -3.69 & 1.92 & 206 & 13 & 9 & 1095.852153 & \\
\hline HR8628 & 2.01 & 0.75 & 188 & 34 & 6 & 1292.41802 & $\mathrm{c}$ \\
\hline HR8634 & 8.71 & 3.07 & 129 & 8 & 192 & 2467.214178 & $\mathrm{a}$ \\
\hline HR8651 & -14.00 & 1.30 & 142 & 15 & 13 & 934.67221 & $\ldots$ \\
\hline HR8682 & -12.53 & 1.85 & 295 & 25 & 15 & 462.639352 & $\ldots$ \\
\hline HR8758 & -18.58 & 1.32 & 318 & 32 & 9 & 441.771597 & $\ldots$ \\
\hline HR8781 & 0.11 & 1.63 & 131 & 9 & 29 & 1541.864433 & \\
\hline HR879 & 14.88 & 1.35 & 181 & 13 & 16 & 707.010613 & $\ldots$ \\
\hline HR8808 & -24.83 & 1.5 & 84 & 42 & 3 & 0.001852 & $\mathrm{c}, \mathrm{d}$ \\
\hline HR8936 & 13.89 & 1.74 & 178 & 10 & 12 & 494.629503 & $\ldots$ \\
\hline HR894 & -15.69 & 1.5 & 207 & 53 & 3 & 0.001585 & $\mathrm{c}, \mathrm{d}$ \\
\hline HR8976 & -11.87 & 1.53 & 204 & 24 & 21 & 1289.321494 & \\
\hline
\end{tabular}


Table 5

(Continued)

\begin{tabular}{|c|c|c|c|c|c|c|c|}
\hline$\underline{\text { Star }}$ & Abs. RV $\left(\mathrm{km} \mathrm{s}^{-1}\right)$ & $\sigma_{R V}\left(\mathrm{~km} \mathrm{~s}^{-1}\right)$ & $V \sin (I)\left(\mathrm{km} \mathrm{s}^{-1}\right)$ & $\sigma_{V \sin (I)}\left(\mathrm{km} \mathrm{s}^{-1}\right)$ & $N_{\text {obs }}$ & $T_{\text {baseline }}$ (days) & Notes \\
\hline HR8988 & -6.14 & 1.5 & 157 & 46 & 3 & 0.001181 & $c, d$ \\
\hline HR899 & 20.54 & 1.5 & 127 & 63 & 4 & 81.794676 & c \\
\hline HR9071 & -9.98 & 1.5 & 153 & 34 & 6 & 901.44463 & $\mathrm{c}$ \\
\hline HR9098 & 10.65 & 0.96 & 198 & 21 & 19 & 592.253391 & $\ldots$ \\
\hline HR932 & 10.23 & 1.5 & 186 & 43 & 3 & 0.00118 & $c, d$ \\
\hline HR954 & 21.24 & 1.5 & 78 & 15 & 7 & 17.997014 & $\mathrm{c}$ \\
\hline HR980 & 2.58 & 0.73 & 301 & 38 & 9 & 25.973321 & $\ldots$ \\
\hline
\end{tabular}

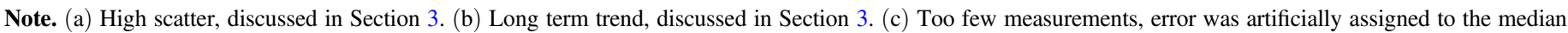
value for our method. (d) Too short time baseline, error was artificially assigned to the median value for our method.

method could be used for observations of young stars as well as those of massive stars.

\section{SUMMARY}

We have developed a method to extract RV measurements from A- and B-type stellar spectra using a forward modeling approach that simultaneously fits the star's RV with the echelle spectrograph's blaze function. Our technique utilizes an extrapolation of the wavelength solution from the iodine calibration region and derives an RV measurement from the entire spectrum simultaneously. This method makes use of qualities of the spectra that are usually weaknesses in RV work, namely the broad spectral lines and multiple featureless orders, to instead serve as strengths in the fitting process. Fitting RVs in this non-traditional manner allows for the analysis of echelle spectra of rapidly rotating stars, which cannot be processed with traditional pipelines.

We found that with our technique, we attain a precision of $1.0 \mathrm{~km} \mathrm{~s}^{-1}\left(0.5-2.0 \mathrm{~km} \mathrm{~s}^{-1}\right)$ for relative RVs. For absolute RVs, which rely upon PHOENIX stellar model spectra as RV templates, the precision is a bit worse $\left(1.5 \mathrm{~km} \mathrm{~s}^{-1}\right)$.

We detect several sources with a high degree in scatter between successive RV measurements taken over the course of anywhere from one to $6 \mathrm{yr}$. Since these stars were observed as calibrators and not science targets, they are often significantly under-sampled. The sparse sampling of each individual target limits our ability to totally characterize these detections. We also detect two significant long-term RV trends (HR 5867 and HR 8028), and redetect a previously known astrometric binary (HR 3067). Two detections have RV time series with slopes that cannot be attributed to any currently known companion in the system (HR 5867) or nearby speckle star (HR 8028). Though we only see an unknown fraction of the phase in our time series, we compute the minimum masses of these new potential companions to be 0.37 and $0.70 M_{\text {odot }}$, respectively.

We thank Emily Rauscher for her careful review of the manuscript and helpful suggestions. J.B. thanks Philip Muirhead for useful conversations and Iryna Butsky for useful comments on the manuscript. We thank the referee, Davide Gandolfi, for his extremely helpful suggestions that led to a vastly improved paper, as well as his suggestions for future directions to take this work. J.B. and A.V. are supported by the National Science Foundation Graduate Research Fellowship, Grants No. DGE 1256260 and DGE 1144152, respectively. J.B. would like to thank Mr. and Mrs. Kenneth Adelman for providing funding for her 2012 Alain
Porter Memorial SURF Fellowship, during which this research was begun. J.A.J. is supported by generous grants from the David and Lucile Packard and Alfred P. Sloan Foundations. This research has made use of NASA's Astrophysics Data System, the SIMBAD database and VizieR catalog access tool, operated at CDS, Strasbourg, France. The data presented herein were obtained at the W.M. Keck Observatory, which is operated as a scientific partnership among the California Institute of Technology, the University of California and the National Aeronautics and Space Administration. The Observatory was made possible by the generous financial support of the W.M. Keck Foundation. The authors wish to recognize and acknowledge the very significant cultural role and reverence that the summit of Mauna Kea has always had within the indigenous Hawaiian community. We are most fortunate to have the opportunity to conduct observations from this mountain.

\section{APPENDIX A EXPECTED PRECISION}

The theoretical best precision on an RV measurement depends on the $\mathrm{S} / \mathrm{N}$ of the measurement, the typical width and depth of spectral features, and the number of spectral features used to calculate the Doppler shift. Butler et al. (1996) derives the expected RV precision:

$$
\sigma_{V}=\left[N_{\text {line }} \sum_{i}\left(\frac{d \mathcal{I}_{i} / d V}{\epsilon_{i}}\right)^{2}\right]^{-1 / 2} \approx \frac{1}{S \sqrt{N_{\text {pix }} N_{\text {line }}}} \frac{\Delta V}{\Delta \mathcal{I}}
$$

when $\epsilon_{i}=\frac{\sqrt{N_{\text {photons }}}}{N_{\text {photons }}}, N_{\text {lines }}$ is the number of spectral lines, $N_{\text {pix }}$ is the number of pixels across which each line occurs, $\Delta \mathcal{I}$ is the relative intensity depth of the spectral features, $\Delta V$ is the average range in wavelength across which this intensity depth occurs, and $S$ is the signal to noise ratio.

For a solar-type spectrum, a typical line might encompass six pixels, with an overall line width of $d V=2.5 \mathrm{~km} \mathrm{~s}^{-1}$ and a relative intensity depth of $d \mathcal{I}=0.2$. With a typical observation $\mathrm{S} / \mathrm{N}$ of 200 and $N_{\text {lines }}=100$ spectral lines, this leads to an expected RV precision of $3.6 \mathrm{~m} \mathrm{~s}^{-1}$ Butler et al. (1996).

To calculate the best-case precision for our method, we calculate the precision for a star in our sample with the median amount of broadening. For such a star, we estimate the relative intensity depth to be roughly equivalent to that for a low-mass star, $d \mathcal{I}=0.2$ (see Figure 1 for a visualization of the comparison). The average line in such a star achieves this intensity dip over $\Delta V=800 \mathrm{~km} \mathrm{~s}^{-1}$ and $N_{\text {pix }}=500$ pixels, 
and has $N_{\text {lines }}=10$ of these lines. Using these median parameters and a standard $\mathrm{S} / \mathrm{N}$ of 100 , we find that were we to be photon limited, we could expect our average precision to be roughly $0.6 \mathrm{~km} \mathrm{~s}^{-1}$.

\section{APPENDIX B ABSOLUTE RVs}

In Table 5, we present derived radial velocity and $V \sin I$ measurements for each star in our sample of A- and B-type calibrators.

\section{REFERENCES}

Abt, H. A., Barnes, R. C., Biggs, E. S., \& Osmer, P. S. 1965, ApJ, 142, 1604 Asplund, M., Grevesse, N., Sauval, A. J., \& Scott, P. 2009, ARA\&A, 47, 481 Bonnell, I. A., \& Bate, M. R. 2005, MNRAS, 362, 915

Bouchy, F., Hébrard, G., Udry, S., et al. 2009, A\&A, 505, 853

Butler, R. P., Marcy, G. W., Williams, E., et al. 1996, PASP, 108, 500

Butler, R. P., Marcy, G. W., Fischer, D. A., et al. 1999, ApJ, 526, 916

Butler, R. P., Vogt, S. S., Marcy, G. W., et al. 2004, ApJ, 617, 580

Campbell, B., Walker, G. A. H., Johnson, R., et al. 1981, Proc. SPIE, 290, 215

Chini, R., Hoffmeister, V. H., Nasseri, A., Stahl, O., \& Zinnecker, H. 2012, MNRAS, 424, 1925

Chubak, C., Marcy, G., Fischer, D. A., et al. 2012, arXiv:1207. 6212

Collins, K. A., Eastman, J. D., Beatty, T. G., et al. 2014, AJ, 147, 39

Cosentino, R., Lovis, C., Pepe, F., et al. 2012, Proc. SPIE, 8446, 84461V

Crane, J. D., Shectman, S. A., \& Butler, R. P. 2006, Proc. SPIE, 6269, 626931

Crane, J. D., Shectman, S. A., Butler, R. P., et al. 2010, Proc. SPIE, 7735, 773553

da Silva, L., Girardi, L., Pasquini, L., et al. 2006, A\&A, 458, 609

Dawson, R. I., \& Fabrycky, D. C. 2010, ApJ, 722, 937

de Bruijne, J. H. J., \& Eilers, A.-C. 2012, A\&A, 546, A61

De Rosa, R. J., Patience, J., Wilson, P. A., et al. 2014, MNRAS, 437, 1216

Dumusque, X., Pepe, F., Lovis, C., et al. 2012, Natur, 491, 207

Duquennoy, A., \& Mayor, M. 1991, A\&A, 248, 485

Fischer, D. A., \& Marcy, G. W. 1992, ApJ, 396, 178

Foreman-Mackey, D., Hogg, D. W., Lang, D., \& Goodman, J. 2013, PASP, 125,306

Frebel, A. 2010, AN, 331, 474

Galland, F., Lagrange, A.-M., Udry, S., et al. 2005, A\&A, 443, 337

Gontcharov, G. A. 2006, AstL, 32, 759
Goodman, Jonathan., \& Weare, Jonathan. 2010, Communications in Applied Mathematics and Computational Science, 5, 1

Gray, D. F. 1976, The Observation and Analysis of Stellar Photospheres (New York: Wiley-Interscience)

Hauschildt, P. H., Allard, F., \& Baron, E. 1999, ApJ, 512, 377

Horch, E. P., van Altena, W. F., Cyr, W. M., Jr., et al. 2008, AJ, 136, 312

Horch, E. P., Bahi, L. A. P., Gaulin, J. R., et al. 2012, AJ, 143, 10

Howard, A. W., Johnson, J. A., Marcy, G. W., et al. 2010, ApJ, 721, 1467

Huang, W., Gies, D. R., \& McSwain, M. V. 2010, ApJ, 722, 605

Jack, D., Hauschildt, P. H., \& Baron, E. 2009, A\&A, 502, 1043

Johnson, J. A., Marcy, G. W., Fischer, D. A., et al. 2006, ApJ, 647, 600

Johnson, J. A., Fischer, D. A., Marcy, G. W., et al. 2007, ApJ, 665, 785

Knutson, H. A., Fulton, B. J., Montet, B. T., et al. 2014, ApJ, 785, 126

Liu, M. C., Fischer, D. A., Graham, J. R., et al. 2002, ApJ, 571, 519

Malkov, O. Y., Tamazian, V. S., Docobo, J. A., \& Chulkov, D. A. 2012, A\&A, 546, A69

Mason, B. D., Martin, C., Hartkopf, W. I., et al. 1999, AJ, 117, 1890

Mayor, M., \& Queloz, D. 1995, Natur, 378, 355

Mayor, M., Pepe, F., Queloz, D., et al. 2003, Msngr, 114, 20

Mermilliod, J.-C., Mayor, M., \& Udry, S. 2009, A\&A, 498, 949

Muirhead, P. S., Vanderburg, A., Shporer, A., et al. 2013, ApJ, 767, 111

Nidever, D. L., Marcy, G. W., Butler, R. P., Fischer, D. A., \& Vogt, S. S. 2002, ApJS, 141, 503

Press, W. H., Teukolsky, S. A., Vetterling, W. T., \& Flannery, B. P. 2002, Numerical Recipes in C++: the Art of Scientific Computing, Vol. 28 (3rd ed.; Cambridge: Cambridge Univ. Press)

Raghavan, D., McAlister, H. A., Henry, T. J., et al. 2010, ApJS, 190, 1

Schwab, C., Spronck, J. F. P., Tokovinin, A., \& Fischer, D. A. 2010, Proc. SPIE, 7735, 77354G

Shaya, E. J., \& Olling, R. P. 2011, ApJS, 192, 2

Spronck, J. F. P., Fischer, D. A., Kaplan, Z. A., Schwab, C., \& Szymkowiak, A. 2013, PASP, 125, 511

Stumpff, P. 1980, A\&AS, 41, 1

Torres, G. 1999, PASP, 111, 169

Valenti, J. A., Butler, R. P., \& Marcy, G. W. 1995, PASP, 107, 966

van Leeuwen, F. 2007, A\&A, 474, 653

van de Kamp, P., \& Vyssotsky, A. 1929, PA, 37, 16

Vogt, S. S., Allen, S. L., Bigelow, B. C., et al. 1994, Proc. SPIE, 2198, 362

Wright, J. T., Marcy, G. W., Fischer, D. A., et al. 2007, ApJ, 657, 533

Wright, J. T., \& Howard, A. W. 2009, ApJS, 182, 205

Wright, J. T., \& Eastman, J. D. 2014, PASP, 126, 838

Zinnecker, H., \& Yorke, H. W. 2007, ARA\&A, 45, 481

Zwahlen, N., North, P., Debernardi, Y., et al. 2004, A\&A, 425, L45 